\title{
Luminosity Functions from the 2dF Galaxy Redshift Survey
}

\author{
Matthew Colless ${ }^{\mathrm{A}}$ \\ A Anglo-Australian Observatory, Epping NSW 1710, Australia. Email: colless@aao.gov.au
}

Received 2004 April 26, accepted 2004 May 25

\begin{abstract}
A summary of the main results from the 2dF Galaxy Redshift Survey of over 221000 galaxies on the galaxy luminosity function and its dependence on surface brightness, spectral type, environment, and local density.
\end{abstract}

Keyword: galaxies: luminosity function

\section{The Survey}

The 2dF Galaxy Redshift Survey (2dFGRS) obtained spectra and measured redshifts for over 221000 galaxies. The survey covered two declination strips, one in the southern Galactic hemisphere spanning $80^{\circ} \times 15^{\circ}$ around the South Galactic Pole (the SGP strip), and the other in the northern Galactic hemisphere spanning $75^{\circ} \times 10^{\circ}$ along the celestial equator (the NGP strip); in addition, there were $992 \mathrm{dF}$ fields scattered over the southern Galactic cap. In total, the survey covers approximately $1800 \mathrm{deg}^{2}$ and has a median redshift depth of $z=0.11$. The 2dFGRS Final Public Release contains positions, magnitudes, redshifts, images, and spectra for all the galaxies in the survey, and is available at www. mso. anu . edu. au/2 dFGRS.

The source catalogue for the $2 \mathrm{dFGRS}$ was a revised and extended version of the APM galaxy catalogue (Maddox et al. 1990), which was created by scanning the photographic plates of the UK Schmidt Telescope Southern Sky Survey. The survey targets were chosen to be galaxies with extinction-corrected magnitudes brighter than $b_{J}=19.45$, although subsequent refinements of the photometry mean that the precise survey limit varies slightly with position. The final calibration of the photographic magnitudes used all available CCD and 2MASS photometry (Jarrett et al. 2000). The 2dFGRS photometry compares well with SDSS photometry (Abazajian et al. 2003) in the region where the two surveys overlap there is a small zero-point offset $(0.06 \mathrm{mag})$ and a surface brightness dependence, due to the difference between the isophotal 2dFGRS magnitudes and the pseudo-total SDSS magnitudes (a variation of 0.5 mag over a range of 4 mag in surface brightness). The RMS scatter between the 2dFGRS and SDSS magnitudes is $0.16 \mathrm{mag}$, or $0.12 \mathrm{mag}$ after removing the surface brightness effect.

The variation with position on the sky of both the limiting magnitude and the magnitude-dependent redshift completeness were carefully determined, and are available as pixellated survey masks in the Final Public Release. Full details of the survey observations and database are given by Colless et al. $(2001,2003)$.

\section{The Galaxy Luminosity Function}

The precise determination of the galaxy luminosity function (LF), and its dependence on spectral type and local density, was one of the major goals of the 2dFGRS (Colless 1999). Several papers have been published by the 2 dFGRS team addressing aspects of these questions - the overall $b_{J}$ LF was derived by Norberg et al. (2002) and the near-IR LFs by Cole et al. (2001); the bivariate distribution of luminosity and surface brightness was obtained by Cross et al. (2001); the variation of the LF for galaxies of different spectral types was investigated by Folkes et al. (1999) and Madgwick et al. (2002); the LF in clusters was obtained by De Propris et al. (2003); the LF dependence on local density is determined by Croton et al. (2004); and the variation of star-formation rate with environment was studied by Lewis et al. (2002). This paper summarises the main results of these works.

\section{The Optical and Near-Infrared LFs}

Norberg et al. (2002) determined the $z=0 \quad b_{J}$-band galaxy LF, accounting for evolution, photometric errors, and redshift incompleteness. Over the absolute magnitude interval $-16.5>M_{b_{J}}-5 \log _{10} h>-22$, the LF is accurately described by a Schechter function with $M_{b_{J}}-5 \log _{10} h=-19.66 \pm 0.07, \alpha=-1.21 \pm 0.03$, and $\phi^{*}=(1.61 \pm 0.08) \times 10^{-2} h^{3} \mathrm{Mpc}^{-3}$. This implies an integrated luminosity density of $\rho_{L}=(1.82 \pm 0.17) \times$ $10^{8} h L_{\odot} \mathrm{Mpc}^{-3}$ (assuming here, and below, an $\Omega_{\mathrm{M}}=0.3$, $\Omega_{\Lambda}=0.7$ cosmology). The uncertainties include the accuracy of the photometric zero-point, cosmic variance, and uncertainty in the evolutionary corrections. Although the preliminary results from the SDSS appeared to show a significantly different luminosity function (Blanton et al. 2001), a subsequent analysis that correctly allowed for the evolution of the galaxy population found the $2 \mathrm{dFGRS}$ and SDSS LFs to be in good agreement (Blanton et al. 2003a).

Although the 2dFGRS is an optically-selected survey, by matching with the Two Micron All Sky Survey (2MASS) Extended Source Catalogue, Cole et al. (2001) produced a near-IR selected galaxy sample with 17173 
measured redshifts. The LFs in the $J$ and $K_{S}$ bands obtained from this sample are fairly well fitted by Schechter functions with parameters $M_{J}^{*}-5 \log h=$ $-22.36 \pm 0.02, \quad \alpha_{J}=-0.93 \pm 0.04, \quad \phi_{J}^{*}=0.0104 \pm$ $0.0016 h^{3} \mathrm{Mpc}^{-3}$, and $M_{K}^{*}-5 \log h=-23.44 \pm 0.03$, $\alpha_{K}=-0.96 \pm 0.05, \phi_{K}^{*}=0.0108 \pm 0.0016 h^{3} \mathrm{Mpc}^{-3}$ for the 2MASS Kron magnitudes. These fits are consistent with those obtained by Kochanek et al. (2001), after correcting for the difference between isophotal and Kron magnitudes, and also with the results of Bell et al. (2003), although they note that the bias of the 2MASS catalogue against low surface brightness galaxies means that the faint end slope and normalisation may need further small corrections.

The surface brightness distribution of the 2dFGRS sample, on the other hand, shows no evidence that significant numbers of low surface brightness or compact galaxies are missed by the survey. Cole et al. estimate the present-day distributions of $b_{J}-K_{S}$ and $J-K_{S}$ colours as a function of the absolute magnitude and use models of the galaxy stellar populations, constrained by the observed optical and IR colours, to infer the galaxy stellar mass function. Integrated over all galaxy masses, this yields a total mass fraction in stars (in units of the critical mass density) of $\Omega_{\text {stars }} h=(1.6 \pm 0.24) \times 10^{-3}$ for a Kennicutt initial mass function (IMF) and $\Omega_{\text {stars }} h=(2.9 \pm 0.43) \times 10^{-3}$ for a Salpeter IMF. These values are consistent with those inferred from observational estimates of the overall star formation history, provided dust extinction is modest.

\section{Luminosity and Surface Brightness}

The bivariate brightness distribution (BBD) is the extension of the luminosity function that gives the distribution of the population over both luminosity and surface brightness. It allows the measurement of the local luminosity density, $j_{b_{J}}$, and of the galaxy luminosity and surface brightness distributions, while accounting for surface brightness selection biases. The BBD for the 2dFGRS derived by Cross et al. (2001) shows a strong luminositysurface brightness relation, $M_{b_{J}} \propto\left(2.4_{-0.5}^{+1.5}\right) \mu_{\mathrm{e}}$; a similar relation is found in the SDSS by Blanton et al. (2003b). In terms of the number density, the peak of the galaxy population lies at $M_{b_{J}}>-16.0$.

Within the well-defined selection limits $\left(-24<M_{b_{J}}<\right.$ $-16.0 \mathrm{mag}, 18.0<\mu_{\mathrm{e}}<24.5 \mathrm{mag} \operatorname{arcsec}^{-2}$ ) the luminosity density is dominated by the contribution from conventional giant galaxies (90\% of the luminosity density is provided by galaxies with $-22.5<M_{b_{J}}<-17.5$, $18.0<\mu_{\mathrm{e}}<23.0$ ). The luminosity-density peak lies away from the selection boundaries, implying that the 2dFGRS is complete in terms of sampling the local luminosity density, and that luminous low surface brightness galaxies are rare. The best estimate for the local luminosity density, after correcting for surface brightness effects, is $j_{b_{J}}=(2.49 \pm 0.20) \times 10^{8} h L_{\odot} \mathrm{Mpc}^{-3}$. This is an increase of $37 \%$ over the estimate obtained without incorporating surface brightness selection effects, which may therefore explain much of the discrepancy between previous estimates of the local luminosity density.

\section{Variations in the LF with Spectral Type}

The variations in the LF as a function of spectral type have been explored by Folkes et al. (1999) and again, with a larger and better-calibrated dataset, by Madgwick et al. (2002). The spectral types for the galaxies are defined using a linear combination of the first two projections derived from a principal component analysis. This parameter, $\eta$, identifies the average emission- and absorptionline strength in the galaxy spectrum, and hence is a useful indicator of the present star formation rate. The study by Madgwick et al. used 75000 high signal-tonoise ratio, low-redshift galaxies. As the type goes from passive to actively star-forming galaxies there is a systematic steepening of the faint-end slope (from $\alpha=-0.54$ to $\alpha=-1.50$ ), and a corresponding faintening of the characteristic magnitude (from $M_{b_{j}}^{*}-5 \log h=-19.6$ to -19.2). A Schechter function provides a poor fit to the LF of the quiescent types for very faint galaxies $\left(M_{b_{J}}-5 \log h<-16.0\right)$, suggesting the presence of a significant dwarf population.

\section{The LF in Clusters}

The composite LF for galaxies in 60 previously catalogued clusters was obtained from the $2 \mathrm{dFGRS}$ by De Propris et al. (2003). The LF spans the range $-22.5<M_{b_{J}}<$ -15 , and is well fitted by a Schechter function with $M_{b_{j}}^{*}-5 \log h=-20.07 \pm 0.07$ and $\alpha=-1.28 \pm 0.03$. It differs significantly from the field LF, having a characteristic magnitude that is approximately $0.3 \mathrm{mag}$ brighter and a faint-end slope that is approximately 0.1 steeper. There is no evidence for variations in the LF across a wide range of cluster properties: the LF is similar for clusters with high and low velocity dispersions, for rich and poor clusters, for clusters with different Bautz-Morgan types, and for clusters with and without substructure. The core regions of clusters differ from the outer parts, however, in having an excess of very bright galaxies. When the LFs are broken down into early (quiescent), intermediate, and late (star-forming) spectral types, it is found that, as in the field, the LFs of earlier-type galaxies have brighter characteristic magnitudes and shallower faint-end slopes; however, the LF of early-type galaxies in clusters is both brighter and steeper than its field counterpart, although the LF of late-type galaxies is very similar. The trend of faint-end slope with spectral type is therefore much less pronounced in clusters than in the field, explaining why variations in the mixture of types do not lead to significant differences in the cluster LFs. As discussed by De Propris et al. (2003), the differences between the field and cluster LFs for the various spectral types can be qualitatively explained by a combination of the suppression of star formation in the dense cluster environment together with mergers to produce the brightest early-type galaxies. 


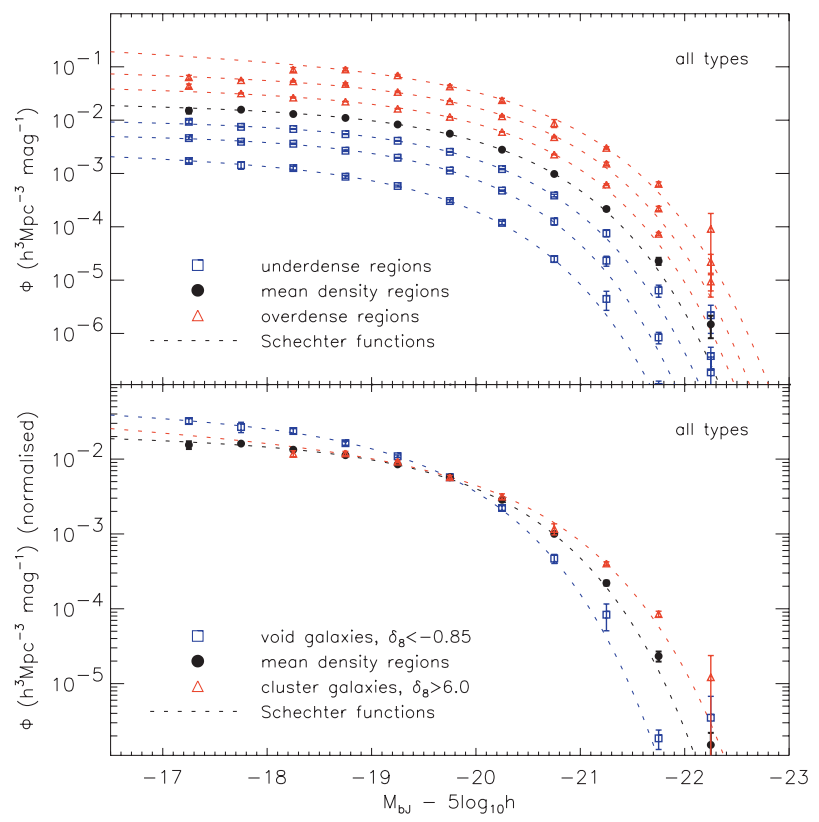

Figure 1 Top: The 2dFGRS luminosity functions in regions of varying density contrast, $\delta_{8}$, from void to mean density to cluster. The best-fit Schechter function parameters for each are over plotted with dotted lines. Bottom: The luminosity function for the void, mean density, and cluster regions normalised to $M_{b_{J}}-5 \log h=-19.75$ so as to highlight the relative differences in the galaxy populations of each.

\section{The Dependence of the LF on Local Density}

A further important topic is the variation of the LF as a joint function of spectral type and local density. Croton et al. (2004) used the 2dFGRS to measure the dependence of the $b_{J}$-band galaxy LF on the large-scale environment, defined by density contrast (for volumelimited subsamples) in spheres of radius $8 h^{-1} \mathrm{Mpc}$, and on spectral type, determined by the $\eta$ parameter. The galaxy populations at both extremes of density differ significantly from that at the mean density (see Figures $1 \& 2$ ). The population in voids is more dominated by late types and shows a significant deficit of galaxies brighter than $M_{b_{J}}-5 \log h=-19.5$ and an excess of fainter galaxies. In contrast, clusters have an excess of very bright $\left(M_{b_{J}}-5 \log h<-21.0\right)$ early-type galaxies, in agreement with previous studies. The luminosity functions measured in all density environments, from voids to clusters, can be approximated by Schechter functions with parameters that vary smoothly with local density, but in a fashion which differs strikingly for early- and late-type galaxies.

\section{Star Formation Rate and Environment}

Addressing the same issue from a different perspective, Lewis et al. (2002) measured the equivalent width of the $\mathrm{H} \alpha$ emission line for 11006 2dFGRS galaxies brighter than $M_{b_{J}}=-19.75-5 \log h$ at $0.05<z<0.1$ in the fields of 17 known galaxy clusters. These measurements are used to trace $\mu^{*}$, the star formation rate normalised to $L^{*}$, as a function of distance from the cluster

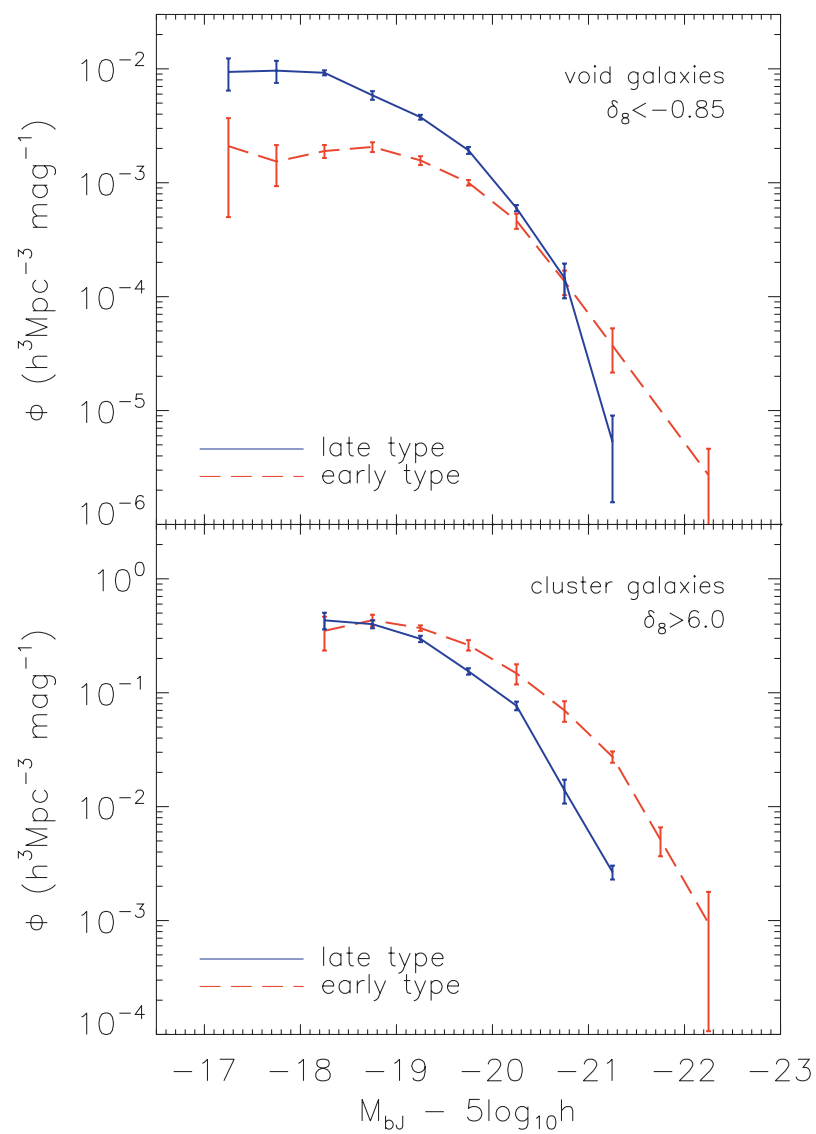

Figure 2 The luminosity function for the (top) void and (bottom) cluster galaxy populations, separated into early and late types. Voids are dominated by late types, although the very brightest void galaxies are early types; in clusters the bright galaxy population is dominated by early types.

centre and local projected galaxy density. The distribution of $\mu^{*}$ steadily skews toward larger values with increasing distance from the cluster centre, converging to the field distribution at distances greater than approximately three times the virial radius. A correlation between star formation rate and local projected density is also found, which is independent of cluster velocity dispersion and disappears at projected densities below about 1 galaxy $\mathrm{Mpc}^{-2}$ (brighter than $M_{b_{J}}=-19.75-5 \log h$ ). This characteristic scale corresponds approximately to the mean density at the cluster virial radius. The same correlation holds for galaxies more than two virial radii from the cluster centre. The environmental influences on galaxy properties are therefore not restricted to cluster cores but are effective in all groups where the density exceeds this critical value. The present-day abundance of such systems, and the strong evolution of this abundance, makes it likely that hierarchical growth of structure plays a significant role in decreasing the global average star formation rate. Finally, the low star formation rates well beyond the virialised cluster rule out severe physical processes, such as ram pressure stripping of disc gas, as being completely responsible for the variations in galaxy properties with environment.

This work has been extended by Balogh et al. (2004) using a combined sample from the 2dFGRS and the SDSS. 
The star-forming and quiescent galaxies (as determined by their $\mathrm{H} \alpha$ equivalent widths) form distinct populations, whose relative numbers vary strongly and continuously with local density on both $1 \mathrm{Mpc}$ and $5 \mathrm{Mpc}$ scales. For the blue-selected 2dFGRS sample, the fraction of starforming galaxies is $70 \%$ in the lowest-density environments and drops to $25 \%$ in the densest clusters. Although the fraction of star-forming galaxies varies with density, the distribution of $\mathrm{H} \alpha$ equivalent widths among the star-forming population is independent of environment. Balogh et al. concluded that the present-day correlation between star formation rate and environment is a result of short-time-scale mechanisms that take place preferentially at high redshift, such as starbursts induced by galaxy-galaxy interactions.

\section{Acknowledgments}

The results described here were obtained by the $2 \mathrm{dF}$ Galaxy Redshift Survey team. The 2dF Galaxy Redshift Survey was made possible through the dedicated efforts of the staff of the Anglo-Australian Observatory, both in creating the $2 \mathrm{dF}$ instrument and in supporting the survey.

\section{References}

Abazajian, K., et al. 2003, AJ, 126, 2081

Balogh, M., et al. 2004, MNRAS, 348, 1355

Bell, E. F., McIntosh, D. H., Katz, N., \& Weinberg, M. D. 2003, ApJS, 149, 289

Blanton, M., et al. 2001, AJ, 121, 2358

Blanton, M., et al. 2003a, ApJ, 592, 819

Blanton, M., et al. 2003b, ApJ, 594, 186

Cole, S., et al. 2001, MNRAS, 326, 255

Colless, M. 1999, RSPTA, 357, 103

Colless, M., et al. 2001, MNRAS, 328, 1039

Colless, M., et al. 2003 (astro-ph/0306581)

Cross, N., et al. 2001, MNRAS, 324, 825

Croton, D., et al. 2004, MNRAS, in press

De Propris, R., et al. 2003, MNRAS, 342, 725

Folkes, S., et al. 1999, MNRAS, 308, 459

Jarrett, T., et al. 2000, AJ, 119, 2498

Kochanek, C., et al. 2001, ApJ, 560, 566

Lewis, I., et al. 2002, MNRAS, 334, 673

Maddox, S., et al. 1990, MNRAS, 243, 692

Madgwick, D., et al. 2002, MNRAS, 333, 133

Norberg, P., et al. 2002, MNRAS, 336, 907 